\title{
Near-Capacity Turbo Trellis Coded Modulation Design
}

\author{
S. X. $\mathrm{Ng}^{1}$, O. Alamri ${ }^{1}, \mathrm{Y} . \mathrm{Li}^{2}$ and L. Hanzo ${ }^{1}$ \\ ${ }^{1}$ School of Electronics and Computer Science, University of Southampton, SO17 1BJ, United Kingdom. \\ Email: \{sxn,ora02r,lh\}@ecs.soton.ac.uk \\ ${ }^{2}$ School of Electrical \& Information Engineering, University of Sydney, Sydney, NSW, 2006, Australia. \\ Email: 1yh@ee.usyd.edu.au
}

\begin{abstract}
Bandwidth efficient parallel-concatenated Turbo Trellis Coded Modulation (TTCM) schemes were designed for communicating over uncorrelated Rayleigh fading channels. A symbol-based union bound was derived for analysing the error floor of the proposed TTCM schemes. A pair of In-phase (I) and Quadrature-phase (Q) interleavers were employed for interleaving the $I$ and $Q$ components of the TTCM coded symbols, in order to attain an increased diversity gain. The decoding convergence of the IQ-TTCM schemes was analysed using symbolbased EXtrinsic Information Transfer (EXIT) charts. The best TTCM component codes were selected with the aid of both the symbol-based union bound and non-binary EXIT charts for the sake of designing capacity-approaching IQ-TTCM schemes in the context of 8PSK, 16QAM and 32QAM signal sets. It will be shown that our TTCM design is capable of approaching the channel capacity within $0.5 \mathrm{~dB}$ at a throughput of $4 \mathrm{bit} / \mathrm{s} / \mathrm{Hz}$, when communicating over uncorrelated Rayleigh fading channels using 32QAM.
\end{abstract}

\section{INTRODUCTION}

Trellis Coded Modulation (TCM) [1] was originally proposed for transmission over Additive White Gaussian Noise (AWGN) channels, but later it was further developed for applications in mobile communications [2]-[4], since it accommodates all the parity bits by expanding the signal constellation, rather than increasing the bandwidth requirement. Turbo Trellis Coded Modulation (TTCM) [5] is a more recent joint coding and modulation scheme that has a structure similar to that of the family of power-efficient binary turbo codes [6], but employs two identical parallel concatenated TCM schemes as component codes. A symbol-based turbo interleaver is used between the two TCM encoders and the encoded symbols of each component code are punctured alternatively for the sake of achieving a higher bandwidth efficiency as detailed in [5], [7]. The design of the TTCM scheme outlined in [5] was based on the search for the best component TCM codes using the so-called 'punctured' minimal distance criterion, where the constituent TCM codes having the maximal 'punctured' minimal distance were sought. However, the TTCM schemes designed for AWGN channels in [5] would exhibit a high error floor, when communicating over Rayleigh fading channels, if any information bits are unprotected by the constituent component codes [8]. Hence, a different TTCM design is needed, when communicating over Rayleigh fading channels.

It was shown in [2] that the maximisation of the minimum Hamming distance measured in terms of the number of different symbols between any two transmitted symbol sequences is the key design criterion for TCM schemes contrived for uncorrelated Rayleigh fading channels, where the fading coefficients change independently from one symbol to another. More specifically, BitInterleaved Coded Modulation (BICM) [9] employing bit-based interleavers was designed for increasing the achievable diversity order to the binary Hamming distance of a code for transmission over uncorrelated Rayleigh fading channels. A parallel-concatenated Turbo

The financial support of the European Union as well as that of the EPSRC UK and the Ministry of Higher Education of Saudi Arabia is gratefully acknowledged.
BICM scheme was designed in [10] and was analysed in [11] when communicating over Rayleigh fading channels, where a lower error floor is attained as a benefit of having a higher minimum Hamming distance. However, bit-interleaved turbo coding schemes have a poorer decoding convergence [12] compared to their symbolinterleaved counterparts due to the associated information loss, when invoking a bit-to-symbol probability conversion during each decoding iteration [13]. Hence, it is desirable to reduce the error floor without using a bit-based interleaver in order to retain the good convergence properties of symbol-interleaved turbo coding schemes.

More specifically, apart from using bit interleavers, the diversity order of a code can be increased with the aid of spatial diversity, frequency diversity and signal space diversity [14]. More explicitly, signal space diversity is obtained by employing two independent channel interleavers for separately interleaving the In-phase (I) and Quadrature-phase $(\mathrm{Q})$ components of the complex-valued encoded signals, combined with constellation rotation. A TCM scheme designed with signal space diversity was proposed in [15]. On the other hand, it was shown in [16] that a diversity gain may also be attained using IQ interleaving alone - i.e. without constellation rotation - in the context of TCM and TTCM schemes. The diversity associated with IQ interleaving alone was referred to as IQ-diversity [16], where the error floor of the IQ-diversity assisted TTCM (IQ-TTCM) schemes was lower than that of conventional TTCM schemes [16]. Hence, we will design new TTCM schemes employing symbol-based turbo interleavers for attaining an early decoding convergence as well as separate I and Q channel interleavers for achieving a low error floor.

Note that turbo codes exhibit low a Bit Error Ratio (BER) in the low to medium Signal to Noise Ratio (SNR) region due to their early decoding convergence. The asymptotic BER performance of a code at high SNR is mainly dominated by its minimum distance. However, the overall BER performance of a code is influenced not only by the minimum distance, but by several distance spectral components, in particular in the medium SNR region [17]-[19]. Hence, the accurate Distance Spectrum [20] analysis has to consider several distance spectral lines, when designing a turbo-style code. Note further that the overall BER performance of a code is determined by both the effective Hamming distance and the effective product distance, when communicating over uncorrelated Rayleigh fading channels [2]. Hence, a two-Dimensional (2D) distance spectrum constituted by both the Hamming distance and product distance has to be evaluated [21]. Recently, a TTCM scheme employing bit-based turbo interleavers was proposed and analysed in [21], where the corresponding union bound of the BER was derived based on the 2D distance spectrum. However, the convergence of the bit-interleaved TTCM of [21] was again inferior compared to the symbol-interleaved TTCM design, despite having a lower error floor. We will derive the BER union bound for TTCM schemes employing symbol-based turbo interleavers in order to analyse their error floor performance.

EXtrinsic Information Transfer (EXIT) charts constitute useful 
tools, when analysing the convergence properties of iterative decoding schemes. They have been invoked for analysing both concatenated binary coding schemes [22] and non-binary coding schemes [23], [24]. As a result, near-capacity codes have been successfully designed by applying an EXIT chart based technique in [25], [26]. The novel contribution of this paper is that we will employ the low-complexity symbol-based EXIT charts proposed in [24] and the corresponding BER union bound of the TTCM schemes in order to design new, near-capacity symbol-interleaved TTCM schemes. More specifically, new generator polynomials are sought for the TCM component codes, based on their decoding convergence and on the error floor performance of the TTCM decoder, rather than on the 'punctured' minimal distance criterion of the TCM component codes defined in [5].

\section{SySTEM MODEL}

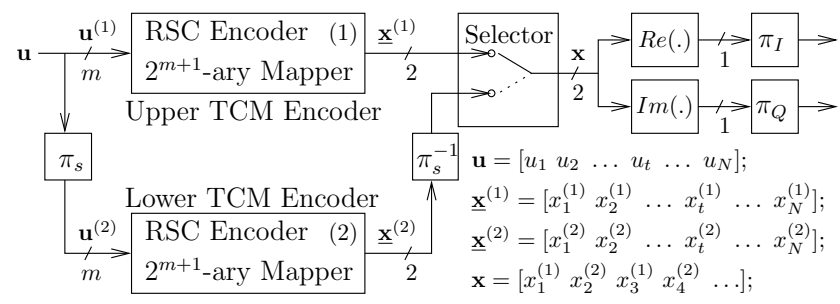

Fig. 1. Schematic of an IQ-TTCM encoder.

In this paper, we consider only two-dimensional TCM and TTCM schemes, where the coding rate is given by $R=m /(m+1)$, employing $2^{m+1}$-ary PSK/QAM signal sets. Hence the effective throughput is $m$ bit per modulated symbol. A TCM encoder consists of a Recursive Systematic Convolutional (RSC) encoder and a signal mapper. An IQ-interleaved TTCM encoder employing two TCM component schemes is shown in Fig. 1. The $N$-symbol uncoded and encoded symbol sequences are denoted as $\mathbf{u}$ and $\mathbf{x}$, respectively. The superscripts (1) and (2) are used for differentiating the uncoded and encoded sequences belonging to the upper and lower TCM encoders, respectively. The I and $\mathrm{Q}$ channel interleavers, namely $\pi_{I}$ and $\pi_{Q}$, are used for independently interleaving the I and Q components of the complex-valued encoded symbol sequence $\mathbf{x}$.

Note that a TTCM scheme employs an Odd-Even Separation (OES) based symbol interleaver $\pi_{s}$ as the turbo interleaver, where odd (even) indexed symbols are mapped to another odd (even) position after interleaving. An OES symbol deinterleaver $\pi_{s}^{-1}$ was also used at the output of the lower TCM encoder. This ensures that after the alternative puncturing, which is performed by the 'Selector' block shown in Fig. 1, all even (odd) indexed symbols of the upper (lower) TCM component encoder are punctured [5]. Note that the information parts of each encoded symbol from the upper and lower TCM encoders before the 'Selector' block are identical. Hence, the information bits are transmitted once and only once, where the resultant TTCM encoded symbol sequence is given by:

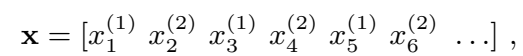

and the odd (even) position symbols are from the upper (lower) TCM component encoder. Note that for simplicity we did not differentiate the sequences before and after the turbo (de)interleaver. More explicitly, we may view the actual encoded symbol sequences transmitted from the upper and lower TCM encoders as:

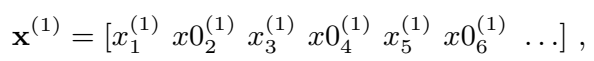

and

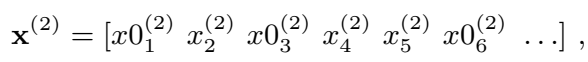

respectively. The notation $x 0$ denotes the punctured TCM symbol, where the parity bit is set to zero.

\section{Symbol-BASEd UnION Bounds}

Let us define the encoded symbol sequence and the erroneously detected symbol sequence of $N$ symbol durations as $\mathbf{x}=$ $\left[\begin{array}{llllll}x_{1} & x_{2} & \ldots & x_{t} & \ldots & x_{N}\end{array}\right]$ and $\hat{\mathbf{x}}=\left[\begin{array}{llllll}\hat{x}_{1} & \hat{x}_{2} & \ldots & \hat{x}_{t} & \ldots & \hat{x}_{N}\end{array}\right]$, respectively. When communicating over uncorrelated Rayleigh fading channels, the Pair-Wise Error Probability (PWEP) of erroneously detecting the sequence $\hat{\mathrm{x}}$ instead of sequence $\mathrm{x}$ can be upper bounded by the following exact-polynomial bound [27, Eq. (35)]:

$$
P_{\operatorname{PWEP}}(\mathbf{x} \rightarrow \hat{\mathbf{x}}) \leq\left(\begin{array}{c}
2 \Delta_{H}-1 \\
\Delta_{H}-1
\end{array}\right)\left(\frac{E_{s}}{N_{0}}\right)^{-\Delta_{H}}\left(\Delta_{P}\right)^{-1}
$$

which is tighter than the Chernoff bound of [2]. More explicitly, $E_{s} / N_{0}$ is the channel SNR, $\Delta_{H}$ is referred to as the effective Hamming distance, which quantifies the diversity order of the code and $\Delta_{P}$ is termed as the effective product distance, which quantifies the coding advantage of a code. More specifically, the product distance of a TCM code is defined as:

$$
\Delta_{P}=\Delta_{P}(\mathbf{x}, \hat{\mathbf{x}})=\prod_{t \in \eta}\left|x_{t}-\hat{x}_{t}\right|^{2},
$$

where $\eta$ represents the set of symbol indices $t$ satisfying the condition of $x_{t} \neq \hat{x}_{t}$, for $1 \leq t \leq N$, while the number of elements in the set $\eta$ is given by $\Delta_{H}=\Delta_{H}(\mathbf{x}, \hat{\mathbf{x}})$, which quantifies the number of erroneous symbol in the sequence $\hat{\mathbf{x}}$, when compared to the correct sequence $\mathbf{x}$.

For the parallel concatenated TTCM scheme, the 'punctured' encoded symbol sequences of the upper and lower TCM encoders, namely $\mathbf{x}^{(1)}$ and $\mathbf{x}^{(2)}$ of Eqs. (2) and (3), respectively, are transmitted at different time instants and hence they are independent of each others. Therefore, the product distance between the TTCM encoded symbol sequences $\mathbf{x}$ and $\hat{\mathbf{x}}$ is given by the product of the individual product distances of the upper and lower TCM-encoded symbol sequences as follows:

$$
\Delta_{P}=\Delta_{P}^{(1)} \cdot \Delta_{P}^{(2)},
$$

where $\Delta_{P}^{(j)}=\Delta_{P}\left(\mathbf{x}^{(j)}, \hat{\mathbf{x}}^{(j)}\right)$ for $j \in\{1,2\}$. Furthermore, the resultant Hamming distance of TTCM is given by the sum of the Hamming distances of the upper and lower TCM codes as:

$$
\Delta_{H}=\Delta_{H}^{(1)}+\Delta_{H}^{(2)}
$$

where $\Delta_{H}^{(j)}=\Delta_{H}\left(\mathbf{x}^{(j)}, \hat{\mathbf{x}}^{(j)}\right)$ for $j \in\{1,2\}$.

The union bound of the average BER of a coding scheme communicating over uncorrelated Rayleigh fading channels can be derived based on [28, p. 125] as:

$$
P_{b} \leq \frac{1}{m} \sum_{\Delta_{P}} \sum_{\Delta_{H}} B_{\Delta_{P}, \Delta_{H}} P_{\mathrm{PWEP}},
$$

where $m$ is the number of information bits per symbol and $B_{\Delta_{P}, \Delta_{H}}$ is the 2D distance spectrum of the code, given by:

$$
B_{\Delta_{P}, \Delta_{H}}=\sum_{w} \frac{w}{N} \cdot A_{w, \Delta_{P}, \Delta_{H}},
$$

where $w$ is the information weight denoting the number of erroneous information bits in an encoded $N$-symbol sequence. Furthermore, $A_{w, \Delta_{P}, \Delta_{H}}$ is the three-dimensional Weight Enumerating Function 
(WEF), quantifying the average number of sequence error events having an information weight of $w$, a product distance of $\Delta_{P}$ and a Hamming distance of $\Delta_{H}$.

\section{A. TCM Distance Spectrum}

Let us derive the WEF $A_{w, \Delta_{P}, \Delta_{H}}$ for a TCM scheme having a block length of $N$ encoded symbols and let the total number of trellis states be $M$. We can define the State Input-Redundancy WEF (SIRWEF) for a block of $N$ TCM-encoded symbols as:

$$
\begin{aligned}
\mathbf{A}(N, S, W, Y, Z)= & \sum_{w} \sum_{\Delta_{P}} \sum_{\Delta_{H}} A_{N, S, w, \Delta_{P}, \Delta_{H}} . \\
& W^{w} Y^{\Delta_{P}} Z^{\Delta_{H}},
\end{aligned}
$$

where $A_{N, S, w, \Delta_{P}, \Delta_{H}}$ is the number of paths in the trellis entering state $S$ at symbol index $N$, which have an information weight of $w$, a product distance of $\Delta_{P}$ and a Hamming distance of $\Delta_{H}$. The notations $W, Y$ and $Z$ represent dummy variables. For each symbol index $t$, the term $A_{t, S, w, \Delta_{P}, \Delta_{H}}$ can be calculated recursively as follows:

$$
A_{t, S, w, \Delta_{P}, \Delta_{H}}=\sum_{S^{\prime}, S: u_{t}} A_{t-1, S^{\prime}, w^{\prime}, \Delta_{P}^{\prime}, \Delta_{H}^{\prime}},(1 \leq t \leq N)
$$

where $u_{t}$ represents the specific input symbol that triggers the transition from state $S^{\prime}$ at index $(t-1)$ to state $S$ at index $t$, while the terms $w, \Delta_{P}$ and $\Delta_{H}$ can be formulated as:

$$
\begin{aligned}
w & =w^{\prime}+i\left(S^{\prime}, S\right), \\
\Delta_{P} & = \begin{cases}\Delta_{P}^{\prime} \cdot \Theta\left(S^{\prime}, S\right), & \text { if } \Theta\left(S^{\prime}, S\right)>0 \\
\Delta_{P}^{\prime}, & \text { else }\end{cases} \\
\Delta_{H} & =\Delta_{H}^{\prime}+\Phi\left(S^{\prime}, S\right),
\end{aligned}
$$

where $w^{\prime}, \Delta_{P}^{\prime}$ and $\Delta_{H}^{\prime}$ are the information weight, the product distance and the Hamming distance, respectively, of the trellis paths entering state $S^{\prime}$ at index $(t-1)$. Furthermore, $i\left(S^{\prime}, S\right)$ is the information weight of symbol $u_{t}$ that triggers the transition from state $S^{\prime}$ to $S$, while $\Theta\left(S^{\prime}, S\right)=\left|x_{t}-\hat{x}_{t}\right|^{2}$ and $\Phi\left(S^{\prime}, S\right) \in\{0,1\}$ are the squared Euclidean distance and Hamming distance between the encoded symbols $\hat{x}_{t}$ and $x_{t}$, where $\hat{x}_{t}$ is the encoded symbol corresponding to the trellis branch in the transition from state $S^{\prime}$ to $S$ and $x_{t}$ is the actual transmitted encoded symbol at index $t$. Let the encoding process commence from state 0 at index 0 and terminate at any of the $M$ possible states at index $N$. Then the WEF used in Eq. (9) is given by:

$$
A_{w, \Delta_{P}, \Delta_{H}}=\sum_{S} A_{N, S, w, \Delta_{P}, \Delta_{H}}
$$

Note that for linear codes [29] or the so-called strong-sense regular TCM schemes of [30], the distance profile of the code is independent of which particular encoded symbol sequence is considered to be the correct sequence. Hence, for the sake of simplicity, we can assume that the all-zero encoded symbol sequence is transmitted, where the union bound of a strong-sense regular TCM scheme [30] can be computed based on Eq (8) using both the PWEP of Eq (4) and the 2D distance spectrum of Eq (9). By contrast, for TCM schemes, which do not satisfy the strong-sense regular definition of [30] we have to consider all possible correct sequences for generating the distance spectrum. Hence a more sophisticated algorithm, such as that proposed in [30] is needed. However, the objective of this paper is not to find the exact union bound of the general TCM or TTCM schemes, but to use the 'approximate' union bound to design near-capacity TTCM schemes. Hence, we will only consider the all-zero encoded symbol sequence as the correct sequence, when computing the union bound. We found that since most TCM and TTCM schemes are not strong-sense regular, applying tailing symbols for having a trellis terminated at state 0 at index $N$ provides a marginal performance improvement compared to having a nonterminated trellis, when communicating over uncorrelated Rayleigh fading channels. Furthermore, the union bound computed based on the exact-polynomial bound of Eq. (4) using the all-zero encoded sequence as the correct sequence turns out to be a very tight bound when approximating the BER performance of various TCM schemes employing 8PSK, 16QAM and 32QAM, as we will demonstrate in Section V.

\section{B. TTCM Distance Spectrum}

Let us now derive the WEF $A_{w, \Delta_{P}, \Delta_{H}}$ introduced in Eq. (9) for a TTCM scheme. Since a TTCM scheme employs two TCM constituent codes, where the parity bits of the upper and lower TCM encoded symbols are punctured at the even and odd symbol indices, respectively, we have to compute two separate distance spectra for the two punctured TCM component codes. Let us denote the SIRWEF of the upper and lower TCM component codes by $\mathbf{A}^{(1)}(N, S, W, Y, Z)$ and $\mathbf{A}^{(2)}(N, S, W, Y, Z)$, respectively. Note that all the punctured parity bits are considered to have a value of ' 0 ' when computing the two SIRWEF terms. We also assume that no termination symbols are used since their performance benefits were found to be modest. Hence both the trellises may be terminated in any of the $M$ possible trellis states. Then we may compute the WEF of the TTCM scheme from the WEF of the two punctured TCM component codes as:

$$
A_{w, \Delta_{P}, \Delta_{H}}=A_{w, \Delta_{P}^{(1)}, \Delta_{H}^{(1)}}^{(1)} \cdot A_{w, \Delta_{P}^{(2)}, \Delta_{H}^{(2)}}^{(2)} \cdot P_{o e}^{N, w},
$$

where $\Delta_{P}=\Delta_{P}(\mathbf{x}, \hat{\mathbf{x}})$ and $\Delta_{H}=\Delta_{H}(\mathbf{x}, \hat{\mathbf{x}})$ are defined in Eqs (6) and (7), respectively. The term $P_{o e}^{N, w}$ in Eq (16) denotes the probability of occurrence for all the associated error events having $w$ information bit errors, when employing an OES symbol interleaver having a length of $N$ symbols. Note that this term equals $1 /\left(\begin{array}{l}N \\ w\end{array}\right)$, when a bit-based random interleaver of length $N$ scrambling 1-bit symbols is employed as the turbo interleaver, as in [31].

Let us now introduce the uniform OES symbol interleaver concept, which evolved from the uniform bit interleaver proposed in [31]. More specifically, an OES symbol interleaver may be partitioned into two symbol interleavers, where the number of bits per symbol equals the number of information bits per symbol, namely $m$, since we are only concerned with the information bit errors as in [31]. The uniform OES symbol interleaver may be defined as in Definition 1.

Definition 1: A uniform OES symbol interleaver of length $N$ symbols is a probabilistic device, which maps a given input sequence of length $N$ symbols having an information weight of $w$ bits into all possible combinations in the odd and even partitions of the interleaver, with equal probability of $P_{o e}^{N, w}$ given by:

$$
P_{o e}^{N, w}=\sum_{\substack{\left.w_{o}=0 \\ w_{o}+w_{e}=w\right)}}^{w_{o}=w} P_{m}^{\lceil N / 2\rceil, w_{o}} \cdot P_{m}^{\lfloor N / 2\rfloor, w_{e}},
$$

where $w_{o}$ and $w_{e}$ are the number of bit errors in the odd and even partitions of the OES symbol interleaver and the term $P_{m}^{L, y}$ denotes the probability of occurrence for the error event having $y$ information bit errors when employing a uniform symbol interleaver of length $L$ symbols, where $L \in\{\lceil N / 2\rceil,\lfloor N / 2\rfloor\}$ and $m$ is the number of bits per symbol. More explicitly, we have:

$$
P_{m}^{L, y}=\frac{1}{\sum_{z \in \chi(y, m)}\left(\begin{array}{l}
L \\
z
\end{array}\right)},
$$


where the set $\chi(y, m)$ consists of all possible combinations of the $z$ number of symbol errors for a given number of bit errors $y$ in a sequence of $L$ symbols.

\section{Code SEARCh Algorithm}

A code-search algorithm designed for finding the TCM constituent codes using the symbol-based EXIT charts of [24] was presented in detail in [32]. Note that the best code selected based on the EXIT charts exhibits the best decoding convergence, but not necessarily the lowest error floor. Hence, we appropriately modified the algorithm of [32] to search for the top 10 polynomials. Then, we choose the one which exibits an error floor lower than the target error floor, possibly at the cost of a slightly worse decoding convergence compared to the best code found from the EXIT charts alone.

\section{RESUltS AND DisCUSSIONS}

The TCM constituent codes found for IQ-TTCM schemes when communicating over uncorrelated Rayleigh fading channels were tabulated in Tab. I for 8PSK, 16QAM and 32QAM signal sets. The EXIT chart based estimation and the simulation based $E_{b} / N_{0}$ threshold values marking the edge of the BER curve's waterfall region were tabulated and compared to the channel capacity limits $\omega$ in the table. The simulation-based threshold corresponds to those $E_{b} / N_{0^{-}}$ values, for which a $\mathrm{BER} \approx 10^{-4}$ is achieved using a block length of 100 '000 symbols.

\begin{tabular}{|c|c|c|c|c|c|}
\hline \multirow{2}{*}{$\begin{array}{l}\text { Modulation/ } \\
\text { States }\end{array}$} & \multirow{2}{*}{$\begin{array}{c}\text { Polynomial (Octal) } \\
{\left[\begin{array}{lllll}g_{r} & g_{1} & g_{2} & g_{3} & \ldots\end{array}\right]}\end{array}$} & \multicolumn{2}{|c|}{ Thresholds (dB) } & \multirow{2}{*}{$\begin{array}{c}\omega \\
(\mathrm{dB})\end{array}$} & \multirow{2}{*}{$\begin{array}{c}m \\
\text { (bit) }\end{array}$} \\
\hline & & Est. & Actual & & \\
\hline 8PSK/4 & $\left.\begin{array}{lllll} & 2 & 4\end{array}\right]$ & $\overline{5.75}$ & $\overline{66.50}$ & 5.38 & 2 \\
\hline $8 \mathrm{PSK} / 8$ & {$\left[\begin{array}{lll}13 & 2 & 4\end{array}\right] *$} & 5.17 & 5.47 & & \\
\hline 16QAM/8 & {$\left[\begin{array}{llll}11 & 2 & 4 & 10\end{array}\right]$} & 8.41 & 8.20 & 7.57 & 3 \\
\hline 16QAM/16 & {$\left[\begin{array}{llll}27 & 2 & 4 & 10\end{array}\right]$} & 8.17 & 8.17 & & \\
\hline 32QAM/16 & {$\left[\begin{array}{lllll}37 & 2 & 4 & 10 & 20\end{array}\right] *$} & 10.03 & 10.20 & 9.98 & 4 \\
\hline 32QAM/32 & {$\left[\begin{array}{lllll}41 & 2 & 4 & 10 & 20\end{array}\right] *$} & 9.90 & 10.20 & & \\
\hline
\end{tabular}

TABLE I

IQ-TTCM CODE POLYNOMIALS FOR UNCORRELATED RAYLEIGH FADING CHANNELS. THE CODES USING POLYNOMIALS MARKED WITH * YIELD A PERFORMANCE LESS THAN 0.5 DB AWAY FROM THE CHANNEL CAPACITY.

Let us now compare the union bound and the actual BER performance of the various TCM and TTCM schemes. Note that we computed a truncated union bound, where only Hamming distances upto $\Delta_{H \text { max }}=4$ and $\Delta_{H \text { max }}=6$ are considered for the TCM and TTCM schemes, respectively. Note that when cosidering a low number of Hamming distances, the truncated union bound for the TTCM scheme will become a lower bound that matches the BER error floor of the TTCM scheme. The number of turbo iterations for the TTCM schemes was fixed to 16. As we can see from Figs. 2, 3 and 4, the estimated union bounds of the 8PSK, 16QAM and 32QAM based TCM schemes exhibit a good match with respect to the corresponding BER curves. As shown in Figs. 2 to 4, the estimated union bounds for the TTCM schemes are lower than the actual TTCM BER curves. However, the TTCM union bounds seemed to have a good match to the IQ-TTCM BER curves in the context of the 8PSK, 16QAM AND 32QAM modulation schemes considered. Hence, we can apply the TTCM union bound to generate a good measure of the expected IQ-TTCM error floor.

As seen from Figs. 2 and 3, the BER performance curves of the 8PSK and 16QAM based TCM schemes are identical, when employing two different code polynomials. By contrast, the BER performance curves of the (IQ-)TTCM schemes are significantly

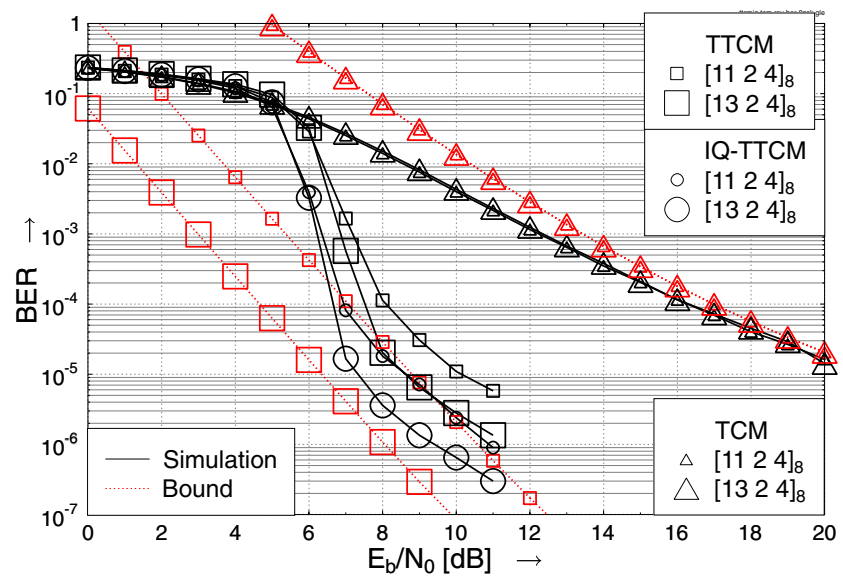

Fig. 2. The BER and union bound performance of the 8PSK-based TCM and (IQ-)TTCM schemes when communicating over uncorrelated Rayleigh fading channels using a block length of $N=1000$ symbols. The product distance spectrum used for generating the union bound was truncated at $\Delta_{P \max }=18$ for both the TCM and TTCM schemes.

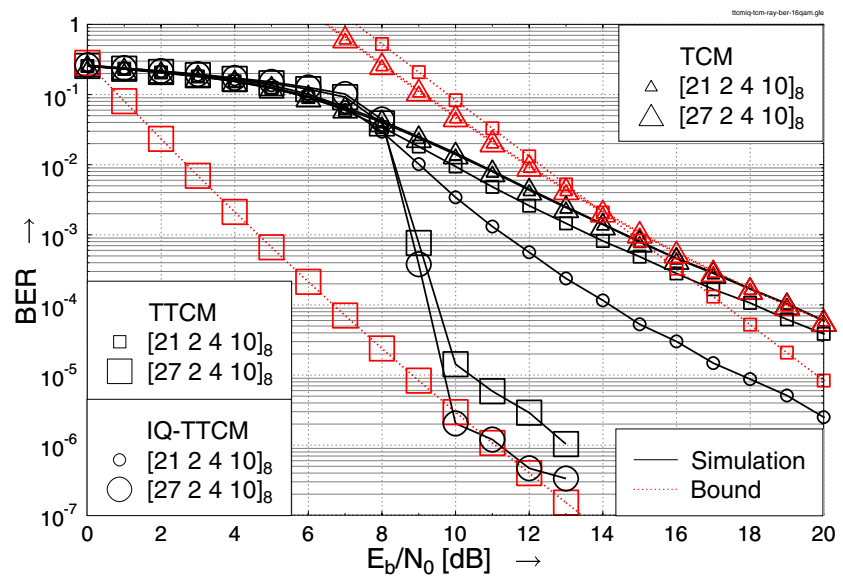

Fig. 3. The BER and union bound performance of the 16QAM-based TCM and (IQ-)TTCM schemes when communicating over uncorrelated Rayleigh fading channels using a block length of $N=1000$ symbols. The product distance spectrum used for generating the union bound was truncated at $\Delta_{P \max }=40$ and $\Delta_{P \max }=60$ for the TCM and TTCM schemes, respectively.

different, when employing two different code polynomials. More explicitly, the 8PSK (IQ-)TTCM scheme performs one dB better at a BER of $10^{-5}$, when employing the proposed code polynomial of $\left[\begin{array}{lll}13 & 2 & 4\end{array}\right]_{8}$ compared to the polynomial of [11 24$]_{8}$ adopted from [5]. We found that the octally represented polynomial [21 2410$]_{8}$, which was designed for a 16QAM TTCM scheme based on the 'punctured' minimal distance criterion of [5] was unable to achieve full decoding convergence due to having a closed tunnel in its EXIT chart. Hence, the BER performance of the 16QAM TTCM scheme employing the proposed polynomial of $\left[\begin{array}{llll}27 & 2 & 4 & 10\end{array}\right]_{8}$ is significantly better than that of the benchmarkers, as it is evidenced in Fig 3.

When we increased the block length to values higher than $N=$ 1000 symbols, the IQ-TTCM schemes exhibited lower error floors and a decoding convergence approaching the estimated thresholds summarised in Tab. I more closely than the scenario using a block length of $N=1000$ symbols, as shown in Figs. 2 to 4. Hence, capacity-approaching TTCM schemes can be successfully designed based on the combined symbol-based EXIT-chart-aided and the truncated union bounding assisted code design proposed. 


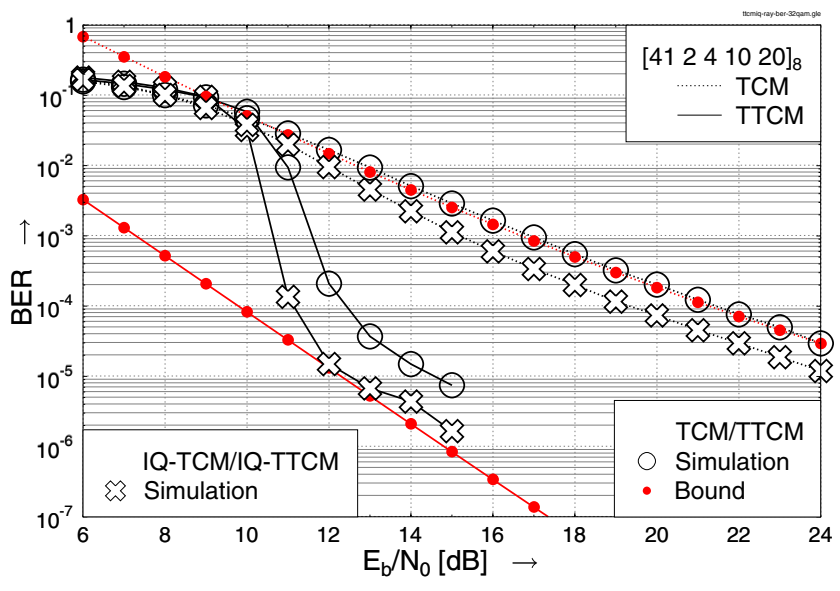

Fig. 4. The BER and union bound performance of the 32QAM-based (IQ)TCM and (IQ-)TTCM schemes when communicating over fast Rayleigh fading channels using a block length of $N=1000$ symbols. The product distance spectrum used for generating the union bound was truncated at $\Delta_{P \max }=50$ and $\Delta_{P \max }=60$ for the TCM and TTCM schemes, respectively.

\section{CONCLUSIONS}

We have designed near-capacity high-order modulation based TTCM schemes by performing a search for good constituent TCM component codes with the aid of symbol-based EXIT charts and truncated symbol-based union bounds. The prime design criterion of capacity-approaching TTCM schemes is that of finding an open tunnel in the corresponding EXIT charts at the lowest possible SNR values, while maintaining a sufficiently low error floor, rather than maximising the 'punctured' minimal distance of the constituent codes [5]. Most of the good constituent codes found assist the TTCM schemes in performing near the channel capacity. Furthermore, the proposed technique may also be employed for designing symbolinterleaved space-time TTCM schemes for approaching the multipleinput multiple-output channel capacity.

\section{REFERENCES}

[1] G. Ungerböck, "Channel coding with multilevel/phase signals," IEEE Transactions on Information Theory, vol. 28, pp. 55-67, January 1982.

[2] D. Divsalar and M. K. Simon, "The design of trellis coded MPSK for fading channel: Performance criteria," IEEE Transactions on Communications, vol. 36, pp. 1004-1012, September 1988.

[3] D. Divsalar and M. K. Simon, "The design of trellis coded MPSK for fading channel: Set partitioning for optimum code design," IEEE Transactions on Communications, vol. 36, pp. 1013-1021, September 1988.

[4] P. Hoeher, "TCM on frequency-selective land-mobile fading channels," in Coded Modulation and Bandwidth-Efficient Transmission, Amsterdam: Elsevier Science Publishers, E. Biglieri and M. Luise (Eds.), pp. 317-328, 1992.

[5] P. Robertson, T. Wörz, "Bandwidth-efficient Turbo Trellis-coded Modulation Using Punctured Component Codes," IEEE Journal on Selected Areas in Communications, vol. 16, pp. 206-218, February 1998.

[6] C. Berrou, A. Glavieux and P. Thitimajshima, "Near Shannon Limit Error-Correcting Coding and Decoding : Turbo Codes," in Proceedings, IEEE International Conference on Communications, pp. 1064-1070, 1993.

[7] L. Hanzo, S. X. Ng, W. Webb and T.Keller, Quadrature Amplitude Modulation: From Basics to Adaptive Trellis-Coded, Turbo-Equalised and Space-Time Coded OFDM, CDMA and MC-CDMA Systems, Second Edition. New York, USA : John Wiley and Sons, 2004.

[8] S. X. Ng, T. H. Liew, L-L. Yang and L. Hanzo, "Comparative Study of TCM, TTCM, BICM and BICM-ID schemes," in IEEE Vehicular Technology Conference, (Rhodes, Greece), pp. 2450-2454, May 2001.

[9] E. Zehavi, "8-PSK trellis codes for a Rayleigh fading channel," IEEE Transactions on Communications, vol. 40, pp. 873-883, May 1992.
[10] S. Le Goff, A. Glavieux and C. Berrou, "Turbo-Codes and High Spectral Efficiency Modulation," in Proceedings of IEEE International Conference on Communications, pp. 645-649, 1994.

[11] T. Duman and M. Salehi, "The Union Bound for Turbo-Coded Modulation Systems over Fading Channels," IEEE Transactions on Communications, vol. 47, pp. 1495-1502, October 1999.

[12] C. Fragouli and R. D. Wesel, "Turbo-encoder design for symbolinterleaved parallel concatenated trellis-coded modulation," IEEE Transactions on Communications, vol. 49, pp. 425-435, March 2001.

[13] B. Scanavino, G. Montorsi, and S. Benedetto, "Convergence properties of iterative decoders working at bit and symbol level," in IEEE Globecom, (San Antonio, TX), pp. 1037-1041, November 2001.

[14] J. Boutros and E. Viterbo, "A power- and bandwidth-efficient diversity technique for the rayleigh fading channel," IEEE Transactions on Information Theory, vol. 44, pp. 1453-1467, July 1998.

[15] B. D. Jelicic and S. Roy, "Design of Trellis Coded QAM for Flat fading and AWGN channels," IEEE Transactions on Vehicular Technology, vol. 44, pp. 192-201, February 1994

[16] S. X. Ng and L. Hanzo, "Space-time IQ-interleaved TCM and TTCM for AWGN and Rayleigh fading Channels," IEE Electronics Letters, vol. 38, pp. 1553-1555, November 2002.

[17] J. Yuan, B. Vucetic and W. Feng, "Combined Turbo Codes and Interleaver Design," IEEE Transactions on Communications, vol. 47, pp. 484-487, April 1999.

[18] W. Feng, J. Yuan and B. Vucetic, "A Code-Matched Interleaver Design for Turbo Codes," IEEE Transactions on Communications, vol. 50, pp. 926-937, June 2002.

[19] S. X. Ng, T. H. Liew, L-L. Yang and L. Hanzo, "Binary BCH Turbo Coding Performance: Union Bound and Simulation Results," in IEEE Vehicular Technology Conference, (Tokyo, Japan), pp. 849-853, May 2000.

[20] Lance C. Perez, Jan Seghers and Daniel J. Costello, "A Distance Spectrum Interpretation of Turbo Codes," IEEE Transactions on Information Theory, vol. 42, pp. 1698-1709, November 1996.

[21] D. Tujkovic, "Unified approach to single- and multiantenna turbo-TCM union bound and constituent code optimization over AWGN and fading channels," in Proceedings of 2004 International Symposium on Personal, Indoor and Mobile Radio Communications, vol. 3, pp. 1618-1622, September 2004.

[22] S. ten Brink, "Convergence behaviour of iteratively decoded parallel concatenated codes," IEEE Transactions on Communications, vol. 49, pp. 1727-1737, October 2001.

[23] A. Grant, "Convergence of non-binary iterative decoding," in Proceedings of the IEEE Global Telecommunications Conference (GLOBECOM), (San Antonio TX, USA), pp. 1058-1062, November 2001.

[24] J. Kliewer, S. X. Ng and L. Hanzo, "On the computation of EXIT characteristics for symbol-based iterative decoding," in 4th International Symposium on Turbo Codes in connection with 6th International ITGConference on Source and Channel Coding, (Munich, Germany), 3-7 April 2006

[25] S. ten Brink, "Rate one-half code for approaching the Shannon limit by 0.1 dB," IEE Electronics Letters, vol. 36, pp. 1293-1294, July 2000.

[26] M. Tüchler and J. Hagenauer, "Exit charts and irregular codes," in Proceeding of the 36th Annual Conference on Information and System Sciences, (Princeton, NJ, USA), March 2002.

[27] S. Siwamogsatham, M. P. Fitz and J. H. Grimm, "A new view of performance analysis of transmit diversity schemes in correlated rayleigh fading," IEEE Transactions on Information Theory, vol. 48, pp. 950-956, April 2002

[28] C. Schlegel, "Chapter 5: Performance Bounds," in Trellis Coding, (New York), pp. 121-151, September 1997.

[29] S. Lin and D. J. Costello, Jr, Error Control Coding: Fundamentals and Applications. Inc. Englewood Cliffs, New Jersey 07632: Prentice-Hall, 1983.

[30] S. Benedetto, M. Mondin and G. Montorsi, "Performance evaluation of trellis-coded modulation schemes," Proceedings of the IEEE, vol. 82, pp. 833-855, June 1994.

[31] Sergio Benedetto and Guido Montorsi, "Unveiling Turbo Codes: Some Results on Parallel Concatenated Coding Schemes," IEEE Transactions on Information Theory, vol. 42, pp. 409-428, March 1996.

[32] S. X. Ng, J. Kliewer, O. Alamri and L. Hanzo, "On the Design of Turbo Trellis Coded Modulation schemes using Symbol-Based EXIT Charts," in IEEE Vehicular Technology Conference, (Montréal, Canada), 25-28 September 2006. 